\title{
Tambo incaico Catarpe Este (informe de avance)
}

A través de un convenio entre la Universidad de Cornell y la Universidad del Norte, se llevó a efecto entre el $1^{\circ}$ de noviembre y el 7 de diciembre de 1976, junto a un grupo de alumnos de dichas universidades y bajo la dirección del autor de este informe, el trabajo de campo en el sitio conocido en la literatura como Catarpe. Aquí detallamos ese trabajo.

\section{Antecedentes}

El sitio Catarpe fue objeto de estudio en el año 1949 por la arqueóloga Dra. Grete Mostny. Su trabajo estuvo orientado a obtener un plano de la distribución espacial de las estructuras, no realizando una excavación de las habitaciones y bodegas. Por las excavaciones de Le Paige en el cementerio de Catarpe, sabemos que éste fue un tambo importante durante el período Inca y culturalmente distinto de otros sitios de la misma época, como es el caso de Solor.

Un reconocimiento del lugar en octubre 24 y 27 nos reveló que el mapeo de la Dra. Mostny y la descripción realizada por ella comprendió sólo una parte del tambo de Catarpe, que ocupa los restos de tres terrazas pleistocénicas y aproximadamente a $40 \mathrm{~m}$ sobre el nivel del río Grande de San Pedro. La ubicación estratégica sobre el valle del río condujo a los incas y a otros pueblos más tempranos, venidos desde su centro cultural en el altiplano boliviano, a realizar un efectivo control sobre todo el movimiento a lo largo del valle, entre el oasis de San Pedro y las alturas andinas. Su posición en una altura prácticamente sin alcance y su fortificación avalaron esta situación. Al mismo tiempo la milicia ocupante y las bodegas de almacenamiento estaban a una distancia cercana a los $10 \mathrm{~km}$ de la concentración indígena en el centro atacameño del actual pueblo de San Pedro. Tales sitios son muy

1 Departamento de Antropología de la Universidad de Cornell, Ithaca, Nueva York, ESTADOS UNIDOS. interesantes para el estudio arqueológico, debido en parte a una reciprocidad cultural, intrusión de artefactos y estilos y a una fluctuación continua de control desde las áreas medulares. La belleza del paisaje de esta planicie cultivable y la constancia de agua fresca fueron otros atractivos.

En el tambo de Catarpe se pueden diferenciar varias formas arquitectónicas que aparentemente se usaron por motivos militares, de almacenamiento y residencias. En cuanto a los depósitos de basuras éstos parece que fueron pocos, pero su conservación es muy adecuada para complementar la información total del sitio.

Los factores arquitectónicos indican una posibilidad de división en sectores. Una parte es predominalmente indígena y probablemente de una larga ocupación (Catarpe Oeste), mientras que la otra (Catarpe Este) es pública y encajona en sentido incásico, con plazas imponentes, bodegas e instalaciones militares. La observación de tales divisiones funcionales y sociales, además de las dificultades de naturaleza mecánica y técnica de trabajo con guijarros y adobes, pisos, fases de construcción y la distribución de basureros en un sitio relativamente grande y compacto, presentará problemas difíciles de interpretación.

Para la realización de nuestro trabajo fue necesario que los alumnos ayudantes Peggy Fischer, Robert Kelly y Julio Sanhueza establecieran su campamento en Catarpe el día 2 de noviembre; días después lo hicieron Kevin Johnston y Olaf Olmos. Desde esta fecha hasta el 5 de diciembre se procedió a excavar en forma continua (alternando en estos días también hicimos una visita al sitio Calarcoco, un campamento precerámico que fuera excavado por Le Paige y Serracino).

Desde el comienzo nuestro trabajo en Catarpe consistió en mapeo, recolección superficial y excavación. Aunque Mostny había publicado un mapa de escala muy reducida de las estructuras medulares 


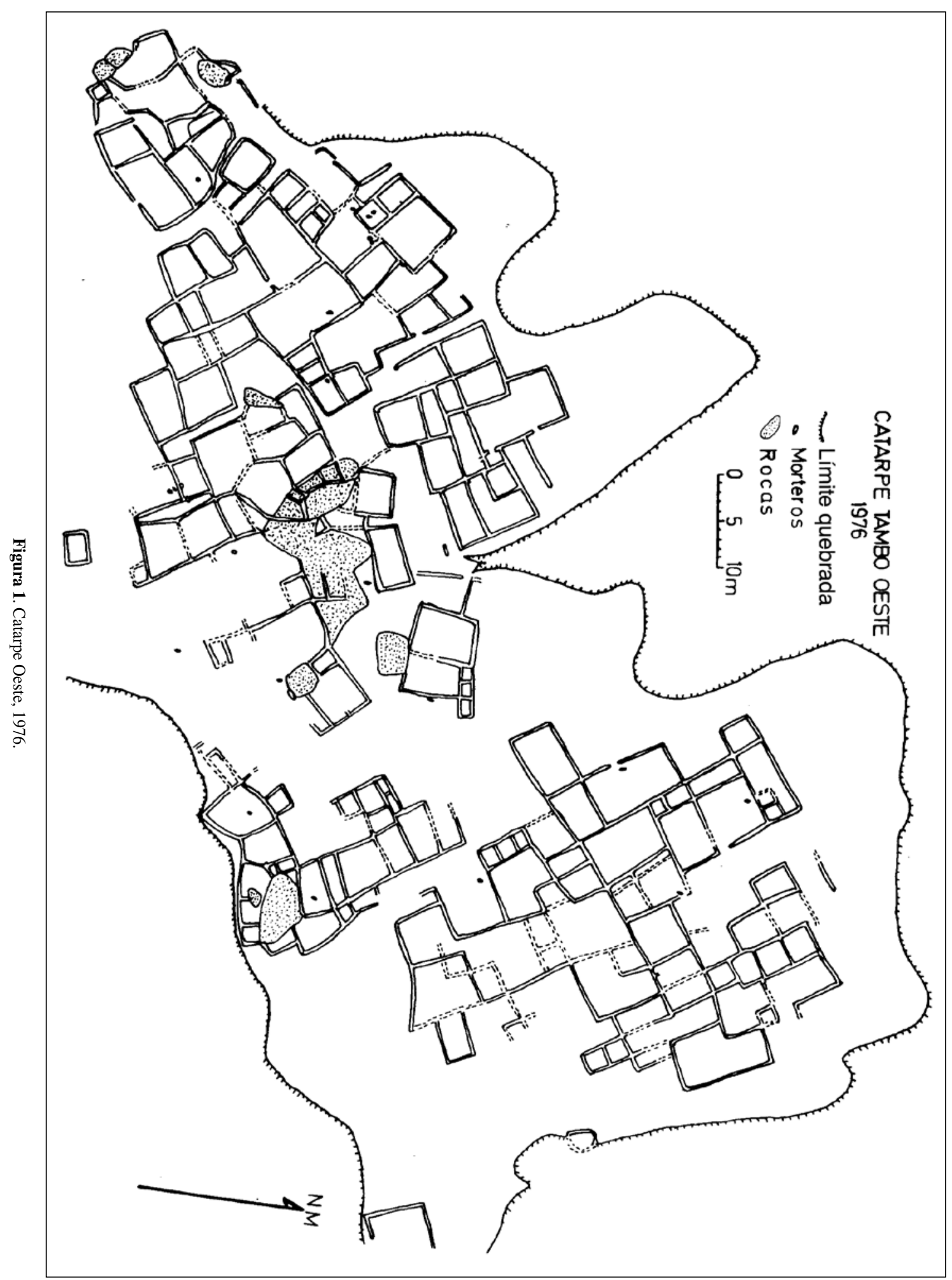




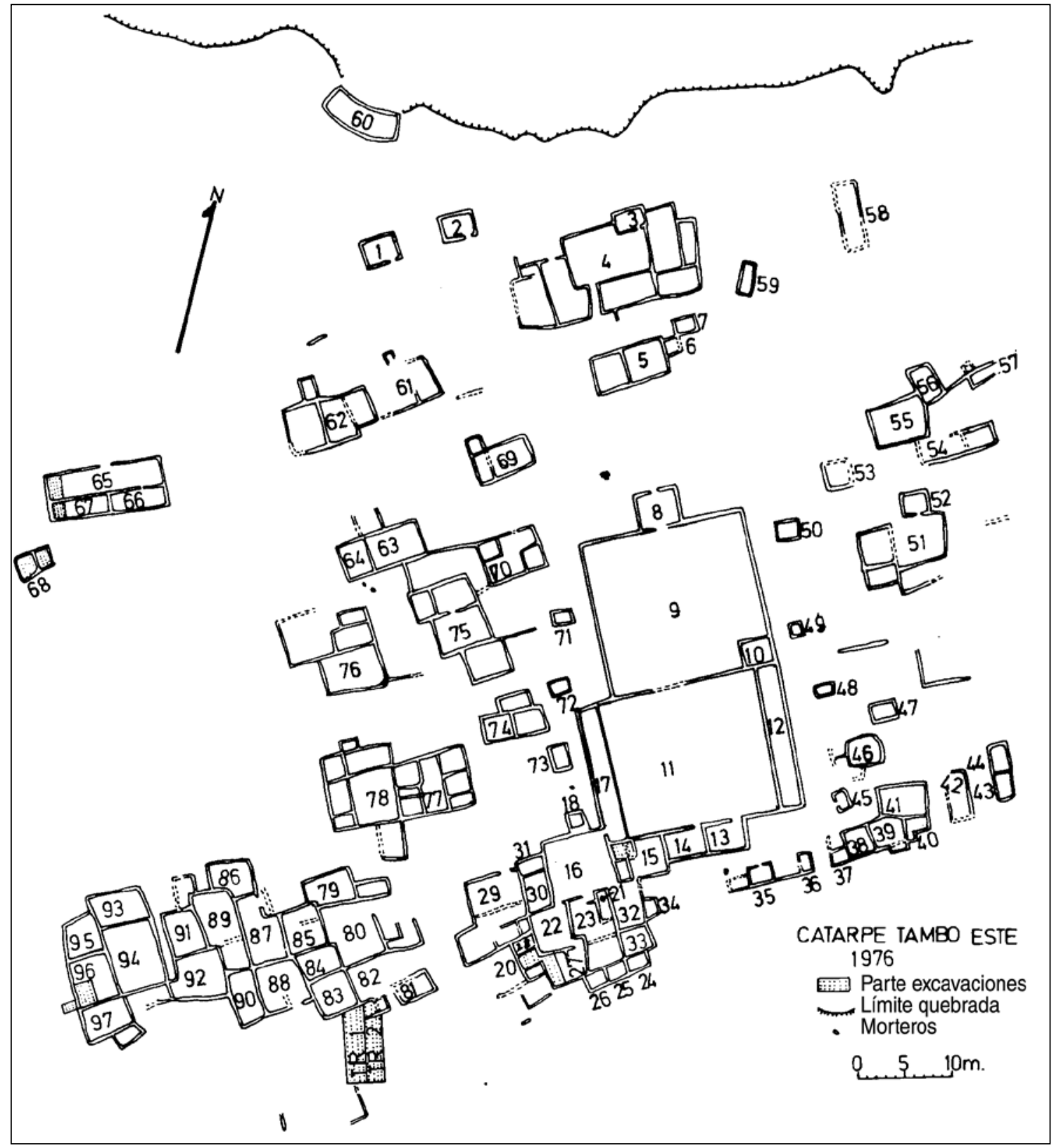

Figura 2. Catarpe Este, 1976. 
de Catarpe Este, decidimos realizar nuestro propio mapeo del sitio entero, por razones de uniformidad y precisión (Figuras 1 y 2). Sin embargo, para evitar confusiones en la literatura, mantuvimos la misma nomenclatura dada por la Dra. Mostny a las 28 estructuras que aparecen en ambos mapas. Hay más o menos 200 recintos u otras estructuras en totalidad, divididos aproximada e igualmente entre este y oeste del tambo, cubriendo un área de 2 ha. Sin la disponibilidad de un taquímetro, el mapa fue hecho por triangulación desde un grid, utilizando huinchas de 25 a $30 \mathrm{~m}$. Dos sectores principales, divididos por una quebrada, fueron fijados en un espacio relativo por medio de acimut leído en una brújula lensática. La triangulación es lenta, pero precisa, especialmente los niveles de terrazas del río. El problema más grande fue la determinación y localización exacta de las paredes caídas, puertas y otros. Es evidente que las estructuras de Catarpe Oeste fueron menos regulares, agrupadas una al lado de la otra y generalmente construidas con menor calidad que en Catarpe Este. Además, algunos morteros quebrados y manos de moler fueron frecuentemente incorporados a las murallas de Catarpe Oeste, indicando una ocupación más larga, como también la presencia de basurales más profundos y más ricos.

Como era el único arqueólogo, decidimos sólo mapear Catarpe Oeste, dejando la recolección superficial y la excavación de aquel sector para campañas futuras. La recolección superficial de Catarpe Este (la parte más formal del sitio) fue realizada por piezas, grupos de cuartos, lo cual resulta mejor que un sistema artificialmente impuesto. Donde había un espacio entre los cuartos los artefactos fueron recolectados en relación a las murallas adjuntas. El perímetro externo al sitio, $10 \mathrm{~m}$ del borde de la terraza, fue también recolectado en forma separada. La recolección superficial ha dado mucha cerámica fragmentada, puntas de proyectil, raspadores, núcleos, guijarros, morteros y también un cincel de cobre. Fragmentos de cerámica de tinajas de almacenamiento predominaban en varias áreas, mientras que en otras los fragmentos de vasijas abiertas fueron más comunes.

La mayor parte de los fragmentos cerámicos se pueden identificar con los tipos ya conocidos en el área de San Pedro, como son: el rojo violáceo, café pulido e inciso. Los tiestos locales del período Inca están bien representados, pero también encontramos tiestos importados con la característica de una pasta de grano fino de color naranja, que podría ser la verdadera inca. Tiestos del tipo Dupont intrusivos fueron también identificados. Ningún tiesto encontrado podría ser anterior al siglo XI. Algunos jarros toscos caracterizados por una base plana $\mathrm{u}$ ocasionalmente por anillo, podrían ser de la época postcolombina, pero prácticamente no encontramos vidrio, hierro u otros materiales europeos en el tambo de Catarpe.

\section{Excavaciones}

Excavamos una trinchera de $2 \times 9 \mathrm{~m}$ a través de un basurero en un declive en la parte sur del sitio. La trinchera 1 se ubica aproximadamente a $25 \mathrm{~m}$ al suroeste de la pieza que la Dra. Mostny denominó con el número 28 y a $15^{\circ}$ oeste del norte. Antes de completar esta trinchera en noviembre 11, tuvimos una idea bastante clara de la base de subsistencia del sector inca de los basureros. Chañar y maíz fueron las evidencias más comunes, pero semillas de algarrobo y zapallo estaban también presentes, como asimismo huesos de auquénidos (presumiblemente llamas o guanacos). Lana de llamas y restos de tejidos se ubicaron frecuentemente en el basural, además se encontraron plumas de colores llamativos, muchas veces mezcladas con los textiles incásicos. En cambio encontramos sólo un fragmento de calabaza pirograbado, un artefacto de madera cuyo uso es desconocido y un cincel de cobre con los mangos de madera. Los artefactos más valiosos que encontramos fueron dos placas de cobre de $10 \mathrm{~cm}^{2}$ y $2 \mathrm{~mm}$ de espesor, con dos figuras muy bien ejecutadas en forma de filigrana, que representan a dos caras humanas con roedores en ambos lados. Las dos placas tenían un prendedor de cobre, talvez para mantenerlos juntos o prendidos a un vestido. No pudimos evitar pensar por qué estos artefactos terminaron en el basural y por qué una de las caras tiene los ojos cerrados y la otra en cambio los representa claramente abiertos.

La trinchera 2, de $2 \mathrm{~m}$ de ancho, extendida paralelamente al este de la trinchera 1, fue un poco menos densa en material. Sin embargo, el registro de material orgánico fue semejante. Nuestro sondeo de este sector de Catarpe terminó con la excavación de la pieza 82-sur, al extremo norte de la trinchera 2 (noviembre 16 y 25). Pudimos ubicar y descubrir como también mapear el piso de este recinto, sobre el cual se encontraba asentada una jarra casi completa y dos herramientas de madera aguzadas. 
La pieza 20, en el extremo suroeste del mapa de Mostny, fue excavada entre el 11 y 26 de noviembre. Es similar a la pieza 82-sur. La pieza 20 fue divivida por una pared no visible desde la parte que no ha sido excavada. La pequeña subdivisión $(1.8$ x $1.1 \mathrm{~m})$ contenía una mayor parte de basuras vegetales y deposiciones posiblemente de cuy. Actualmente tales subdivisiones se hacen en partes rurales de los Andes Centrales para la crianza de cuyes. La parte de mayor espacio en el sector norte de la pieza 20 $(1.9 \times 1.35 \mathrm{~m})$ tiene características que corresponden a una habitación ocupada por el hombre; así lo demuestran los indicadores registrados: un mortero, huesos trabajados, madera y una base plana o mesa sobre la cual ponían artefactos caseros.

Una vez que las paredes fueron despejadas y la pieza excavada hasta el suelo estéril, fue evidente que la pared oriental de la pieza 20 fue una adición que la separaba de la pieza 27, algún tiempo después de la ocupación original. Una trinchera de $1.2 \mathrm{~m}$ de ancho y del largo de la pared occidental de la pieza 27 fue excavada desde noviembre 30 hasta diciembre 5. Se despejó una puerta bloqueada, partes de un piso y partes del techo caído.

La pieza número 25 , ubicada a pocos metros al este de la pieza 27, se pensó que era una bodega debido a su reducido tamaño $(1.95 \times 1.20 \mathrm{~m})$. Investigaciones desde el 17 hasta el 26 de noviembre mostraron desechos abundantes de ocupación humana, incluyendo un guijarro de corte tajante, una mano, pigmento rojo, cucharas quebradas, varias torteras de madera y espinas largas, posiblemente para tejer.

Algunos metros al norte de la pieza 25 excavamos la esquina noroeste de la pieza 15 , que fue internamente subdividida para cerrar un espacio de $2.55 \times 1.75 \mathrm{~m}$. Entre noviembre 26 y diciembre 5 no logramos comprender la secuencia de la construcción de las paredes, concluyendo que este espacio ha sido construido después de la construcción de las plazas 11 y 16 , con las cuales la pieza 15 noroeste se junta por el lado norte y oeste, respectivamente. La entrada en la esquina sureste de la pieza 15 noroeste fue bloqueada algún tiempo antes de que fuera abandonada, pero tal vez lo más interesante de ella fueron las partes inferiores de la pared, elaborada directamente sobre la gravilla estéril de la terraza, lo cual podría ser un resto de una estructura más temprana y totalmente distinta.
Las murallas más altas y gruesas en Catarpe Tambo, que circundan la plaza 11 , miden 22 x $15 \mathrm{~m}$. Optamos por hacer un muestreo en la esquina noreste del cerco con un pozo de $2.3 \times 2.0 \mathrm{~m}$, un lado del cual tenía una pestaña saliente de la muralla norte principal. Esta fue la parte más interesante del registro. En este pozo 12 norte-1, como le designamos, prácticamente despejamos un techo de paja intacto entre noviembre 25 y diciembre 5 . Los soportes de caña fueron también conservados y probablemente corresponden a un palo intacto de la muralla norte. Este techo llegaba a $2.25 \mathrm{~m}$ sobre el nivel del piso, correspondiendo más a la altura de una llama que a la de un hombre. Las partes techadas en los lados este y oeste de la plaza 11 podrían haber servido de corrales, por lo menos en varios intervalos.

Los restos de un techo de paja y cordel de fibra vegetal fino, utilizado para su apoyo, fueron encontrados encima del piso, en la trinchera, al lado oeste de las piezas 65 y 67, excavados entre noviembre 21 y 29. Este complejo de dos piezas estrechas y largas con un patio al frente está aislado de otras estructuras al extremo oeste de Catarpe Este. Aquí se domina una vista excelente de todo el valle de San Pedro. En la pieza 65 encontramos escaso desecho y sólo algunos fragmentos de cerámica que dejamos en el piso. El material orgánico significativo, fuera del techo de paja, lo constituyó una colección de corontas de maíz. Resulta interesante señalar aquí que en otros lugares, especialmente en el basural, las corontas de maíz están asociadas a tallos y hojas; en la pieza 67 solamente se encontraron corontas. Debemos también hacer hincapié que varios fragmentos de cerámica fueron registrados en abundancia en el patio (pieza 65) más que en la pieza 67.

La pieza 96, ubicada al sureste de Catarpe Este, es una de las más grandes estructuras del sector residencial, midiendo casi $5.5 \mathrm{~m}$ de largo y $4.5 \mathrm{~m}$ de ancho. Aquí solamente excavamos el cuadrante suroeste y una trinchera menor al exterior, pero quedamos defraudados al encontrar muy poco material en el relleno. Asimismo la pieza 68-este y oeste, que sospechábamos era una cocina o estructura gruesa, entregó muy poco material.

El día 5 de diciembre terminamos nuestro trabajo de campo, finalizando así la primera etapa del Proyecto Tambo de Catarpe. 
Agradecimientos Quisiera agradecer tanto el aporte financiero como la hospitalidad brindada a nosotros por el rector de la Universidad del Norte, don Hernán Danyau. Asimismo al R. P. Gustavo Le Paige, director del Museo Arqueológico de San Pedro de
Atacama, y al señor George Serracino, investigador del mismo museo. También a los alumnos-ayudantes que trabajaron en este proyecto.

Diciembre 10 de 1976. 


\section{Nota sobre la irrigación en San Pedro de Atacama, II región, norte de Chile (informe de avance)}

BARBARA LYNCH ${ }^{1}$

Los sistemas de irrigación en América del Sur han sido muy raramente estudiados en un contexto social y económico en las comunidades a las cuales ellos sirven. Una excepción notable a esta situación es el estudio que fuera realizado por la CORFO, en el pueblo de San Pedro de Atacama, ubicado al interior de la ciudad de Calama, en la II región del norte de Chile, a una altitud de $2400 \mathrm{~m}$.snm. Este trabajo realizado por la CORFO, a cargo de la profesora Aranda y su equipo (1961-1964), relaciona la irrigación a factores como demografía, fragmentación de propiedades y condiciones de mercado, como también a factores climáticos y geológicos.

Sin embargo, en los 15 años que van desde que la CORFO realizó sus estudios, las estructuras físicas y la organización social de los ríos San Pedro de Atacama y Vilama en su sistema de irrigación han tenido grandes cambios. Entre los años 1963 y 1967, más de $50 \mathrm{~km}$ de canales matrices y secundarios fueron cementados con lajas. Posteriormente otros $30 \mathrm{~km}$ fueron también cementados. Además que nuevas puertas y sifones eran instalados desde el ayllu de Cuchabrachi al comienzo del sistema hasta los canales secundarios que proveen los ayllus cercanos al Salar de Atacama. Sin la eficiente ayuda de la Dirección de Riego, estos cambios no habrían podido llevarse a efecto (Figura 1).

La presencia de personeros de gobierno en San Pedro de Atacama permitió aumentar enormemente la construcción de canales durante el año 1963. Hoy, no obstante, este personal gubernamental ha sido reducido casi al mínimo. Asimismo la Dirección Agrícola Experimental ha cerrado sus puertas y la Dirección de Riego tiene a un operario encargado y a dos empleados en toda la zona. Ahora, en septiembre de 1977, estos operarios, bajo la dirección de la Junta de Riego, deberán tener la responsabilidad del mantenimiento de todo el sistema. Al nivel del

1 Universidad de Cornell, Ithaca, ESTADOS UNIDOS. sistema, los tres niveles descritos por Aranda han sido substituidos por siete grupos, uno por cada canal que tiene permanentemente aguas corrientes. Cada grupo tiene su Junta de Regantes y elige un cuidador para supervisar los canales. Estos cuidadores pagados han sustituido al Inspector de Aguas, que en los años 60 tenía que vigilar todo el sistema. Mas recientemente la Junta Central experimentó un aumento al incluir delegados de cada grupo, que significó una buena medida para el mejor rendimiento entre la comunicación de los grupos y la Junta Central.

Todos estos cambios y sus efectos tendremos todavía que analizarlos en forma sistemática. Es verdad que los mejoramientos físicos han significado una reducción en las pérdidas de aguas y simplificado la limpieza y el mantenimiento de los canales, pero estas ventajas se pierden al fomentarse las preferencias en la distribución del agua. También el corregir y dejar los canales rectos ha significado un sistema rígido, mientras que anteriormente fue un sistema ineficiente pero flexible. La reorganización de los regadores en siete grupos podría ser una bendición impuesta, pero no aceptada. Un gran grupo de propietarios en el sistema de los ríos San Pedro/Vilama poseen tierras en varios grupos. Este patrón de propiedad reduce los conflictos entre los grupos, pero también es difícil encontrar tanta gente voluntaria para manejar siete grupos de cuatro personas cada uno y una Junta Central de 11 miembros.

Una serie de otras preguntas surge cuando se considera el sistema de los ríos San Pedro/Vilama en un contexto de desarrollo regional. En la II región, el Ministerio de Aguas ha optado por dar prioridad a los sistemas de irrigación por considerarlos fuente de desarrollo urbano e industrial. La Dirección de Riego debe prever un papel más importante para San Pedro de Atacama. Más que un simple proveedor de hortalizas para Chuquicamata y Calama, se deben crear condiciones para evitar la emigración de los habitantes de los pueblos cordilleranos, que se mantienen con la agricultura hacia los centros urbanos. Con la disolución de la Dirección de Riego, como 


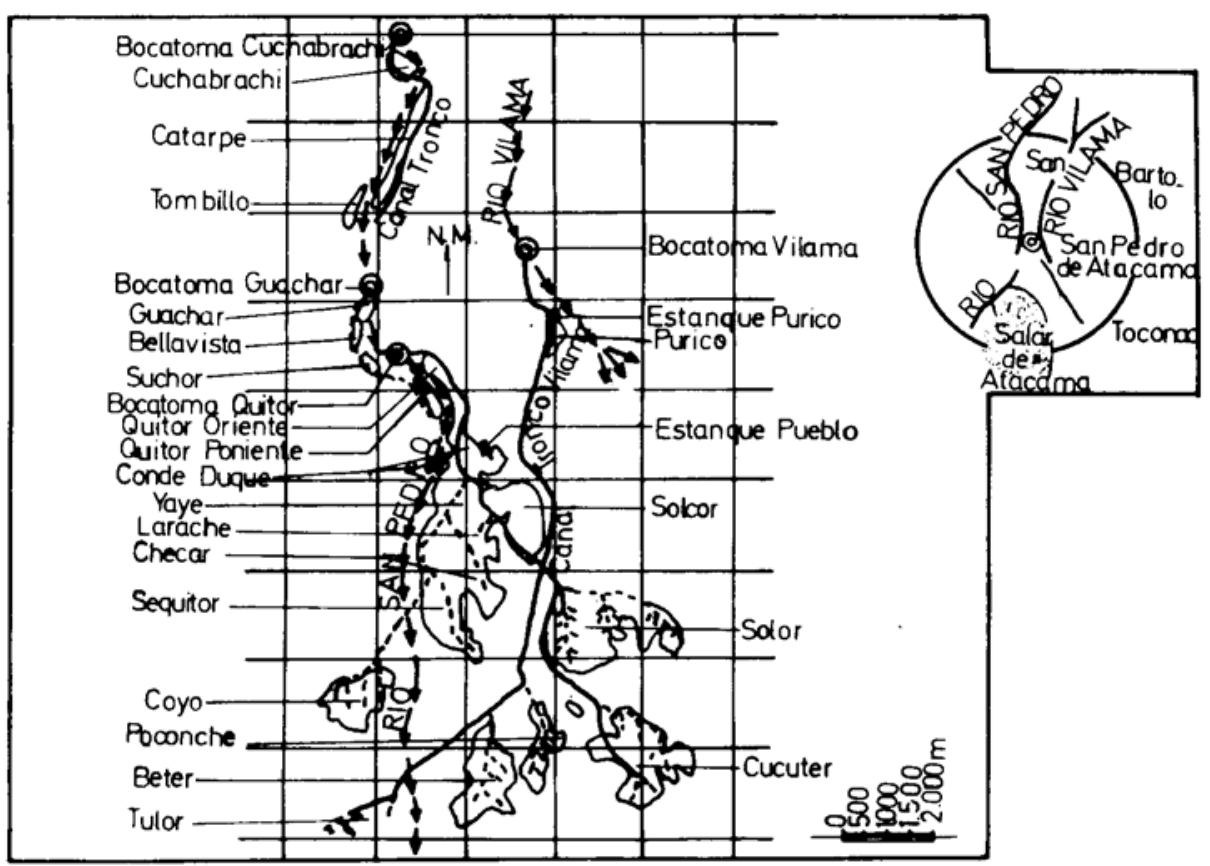

Figura 1. Canales de regadío de San Pedro de Atacama.

departamento de la Dirección de Obras Públicas, es muy probable que la agricultura cercana al Salar de Atacama ceda al desarrollo industrial y la población rural continúe disminuyendo en forma acelerada.

Estos desarrollos han sido complementados con progresos significativos en los estudios de los aspectos sociales de los sistemas de irrigación a escalas menores. Antropólogos sociales han hecho una serie de críticas a la teoría de Wittfogel que sostiene que la autoridad central política ha sido el resultado de la administración de fuentes de agua en tierras áridas (Millon 1962; Mitchell 1973; Hunt y Hunt 1976). Estas críticas han generado, a su vez, un número de estudios sobre sistemas de canales desde los campos de arroz de Asia hasta los Alpes suizos. En ninguna de las comunidades estudiadas, sin embargo, la agricultura es totalmente dependiente del sistema de irrigación como es el caso de San Pedro de Atacama. Es importante ver que la adaptación a la aridez extrema produce una distribución de agua y organización sustancialmente diferente a áreas cercanas y con más agua. Una comparación del sistema de canales de los ríos San Pedro y Vilama con otras comunidades andinas permitiría analizar el papel de las variables ecológicas en sistemas sociales (ver Soler Bustamante 1954: 580; Doughty 1968; Mitchell 1976).

Agradecimientos Gracias a la generosa colaboración y ayuda del R. P. Gustavo Le Paige, director del Museo Arqueológico de San Pedro de Atacama; a George Serracino y a los habitantes de San Pedro de Atacama, como a los oficiales de riego de la región, departamento y grupos, pude abordar e investigar los problemas referidos en esta nota. Fue así que el conjunto de datos sobre demografía, agricultura y uso de aguas me permitió determinar con precisión las relaciones del sistema de irrigación de San Pedro de Atacama y Vilama, como también ver lo provechoso del sistema de irrigación.

Diciembre 10 de 1976. 


\section{REFERENCIAS CITADAS}

ARANDA, X., 1961-64. San Pedro de Atacama, elementos diagnósticos para un plan de desarrollo local. Informaciones Geográficas XI-XIV: 9-61.

DOUGHTY, P., 1968. Huaylas: An Andean district in search of progress. Cornell University Press, Ithaca.

HUNT, E. y R. HUNT, 1976. Canal irrigation and local social organization. Current Anthropology 17: 389-411.

MILLON, R., 1962. Variations in social responses to the practice of irrigation agriculture. En Civilizations in desert lands. R. Woodbury (Ed). Anthropological Papers 62, University of Utah, Salt Lake City.
MITCHELL, W., 1973. The hydraulic hypothesis: A reappraisal. Current Anthropology 14: 522-534.

1976. Irrigation and community in the Central Peruvian Highlands. American Anthropologist 78: 25-43.

SOLER BUSTAMANTE, E., 1954. La agricultura en la comunidad de San Pedro de Huancaire. Revista del Museo Nacional 23: 1-15.

1958. La comunidad de San Pedro de Huancaire. En Las actuales comunidades de indígenas Huarochiri en 1955, J. Matus y otros (Eds.). Universidad de San Marcos, Lima. 


\section{Informe de actividades del Programa Paleoindio año 1976}

LAUTARo NúÑEZ ${ }^{1}$

De acuerdo con los antecedentes de este programa publicados en números anteriores y consecuente con sus objetivos, durante el año 1976, se ha continuado la labor de campo a través de sitios vinculados con ocupaciones correspondientes a los períodos Paleoindio y Arcaico en los territorios áridos y semiáridos de Chile. En lo referente a las tempranas ocupaciones paleoindianas se logró la elaboración de un proyecto específico para el sitio Quereo (Los Vilos), integrando las coautorías del geólogo chileno Juan Varela y del paleontólogo argentino Rodolfo Casamiquela, equipo multidisciplinario que aspira a continuar con estas indagaciones tempranas. Con respecto a los sitios arcaicos, ubicados en los distritos arqueológicos de Tiliviche, Tuina y Tulan, se continuaron ampliando las excavaciones, y por ahora se prevé establecer las últimas campañas en estos sitios en relación a los chequeos radiocarbónicos que deberán establecerse a corto plazo. Paralelamente se han intensificado las muestras de análisis cuantitativo de los desperdicios de los sitios arcaicos antes referidos más los yacimientos de Tambillo y Puripica. En relación a los estudios de taxonomía lítica se alcanzó a preparar la colección de Tulan en términos de limpieza y marcación.

Durante 1976 llegaron las primeras fechas radiocarbónicas que a continuación proporcionamos, sin comentario, por cuanto los estudios monográficos en desarrollo explicarán el significado de cada una de ellas:

- Puripica: $2100 \mathrm{AC}$

- Tulan 52: 2390 y $2320 \mathrm{AC}$

- Tulan 51A: $3040 \mathrm{AC}$

- Tiliviche 1B: 5900 y 4850 AC

- Quereo: $7420 \mathrm{AC}$

La fecha de Puripica correspondería al comienzo de ocupación del sitio clásico descrito por Le Paige. Sin embargo, hemos enviado nuevas muestras para lograr mayor seguridad en la datación de este yacimiento.

1 Universidad del Norte, CHILE.
Los sitios Tulan 52 y Tulan 51A corresponden a nuevos yacimientos ubicados por el programa en la banda sur de la quebrada de Tulan al sur de los yacimientos clásicos descritos por Le Paige. Estas fechas demuestran un tiempo aproximado del comienzo de ocupación de un conglomerado semialdeano precerámico y de un campamento restringido y relacionado a un trabajo de taller. En consecuencia, las fechas recibidas no determinan un tiempo para las industrias descritas por Le Paige, y sin duda alguna que el problema cronológico en el área de Tulan tomará más tiempo, recursos y muestras.

En relación con las dataciones de Quereo (dos similares), éstas proceden de dos sectores distantes de una misma turba que sella el sedimento en donde aparecen los restos de megafauna y presencia humana.

Aunque la cantidad de carbón encontrada en el campamento de Tambillo es mínima, y teniendo en mente que es muy difícil registrar fogones con material adecuado para fechar, la muestra existente será procesada y su resultado se recibirá de un momento a otro. También esperamos la llegada de un experto en fauna del Smithsonian Institution correspondiente a las etapas arcaicas que se conservan en las colecciones del Museo Arqueológico de San Pedro de Atacama.

En el mes de noviembre de 1976 una misión del departamento de antropología del Smithsonian Institution realizó una visita oficial a la Universidad del Norte y al Museo de La Serena, con el fin de evaluar el desarrollo del programa. De esta visita se desprendió que el intercambio de experiencias y los avances de las investigaciones pueden considerarse altamente positivos, ya que incluso se prepararon nuevos proyectos con la participación del geólogo Juan Varela y del paleontólogo Rodolfo Casamiquela, expertos en geología del Cuaternario y fauna extinta, respectivamente. En suma, la continuidad del programa está orientada a dos situaciones bien definidas: 
a) Se avanzará con exploraciones en áreas favorables en torno a tempranas ocupaciones tanto en el Norte Grande y Chico, poniendo énfasis en sitios con dataciones anteriores a los $6000 \mathrm{AC}$. Se trata de definir mejor el contacto Arcaico Temprano con Paleoindio, ya que diversas fases del Período Arcaico se han intercalado adecuadamente, pero se requiere de mayor información bajo el límite temporal antes referido.

b) Intensificar las excavaciones en sitios propiamente paleoindios, para clarificar sus contextos culturales y puntualizar su rango cronológico.
Este programa demuestra un esfuerzo cooperativo de parte del departamento de Antropología del Smithsonian Institution, Museo de La Serena y Universidad del Norte. Por esta misma razón el sensible fallecimiento del Conservador del Museo de La Serena, don Jorge Iribarren, acaecido en enero del año 1977, ha significado una pérdida realmente irreparable.

Antofagasta, marzo de 1977. 